\section{NATURAL KILLER CELLS}

\section{Maintaining tolerance}

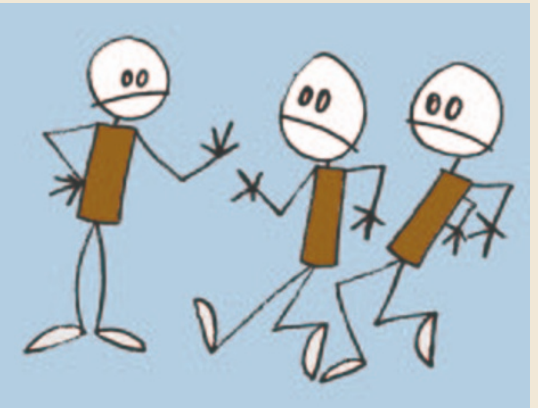

According to the 'missing-self' hypothesis, natural killer $(\mathrm{NK})$-cell tolerance is maintained by the interaction of inhibitory NK-cell receptors with MHC class I molecules expressed at the surface of autologous cells. However, NK cells from MHC-class-I-deficient humans maintain self-tolerance - so how does this occur? Megan McNerney and colleagues now show that there is another system for controlling NK-cell tolerance, in which engagement of 2B4 (also known as CD244) at the surface of mouse NK cells by CD48 at the surface of autologous cells provides a 'stop' signal for NK-cell cytotoxicity.

Previous studies have shown that engagement of 2B4 can inhibit NK-cell responses to tumour cells. In this study, the authors first asked whether both Ly49 molecules and 2B4 (which are inhibitory $\mathrm{NK}$-cell receptors in mice) are required for the inhibition of NK cells or whether either system alone is sufficient. In NK-cell cytotoxicity assays, maximal cytotoxicity was detected in the absence of both inhibitory systems, showing that they are non-redundant for protection against NK-cell cytotoxicity.

So, is $2 \mathrm{~B} 4$ responsible for maintaining NKcell tolerance in the absence of MHC class I expression: for example, in $\beta_{2}$-microglobulin $\left(\beta_{2} \mathrm{~m}\right)$-deficient mice? In cytotoxicity assays carried out in the absence of 2B4-CD48mediated inhibition, $\beta_{2} m^{-/-} \mathrm{NK}$ cells had a higher lytic capacity, indicating that the 2B4-CD48 system normally operates in the absence of MHC class I expression. Similar results were obtained in the reverse situation, using NK cells from C57BL/6 mice that lack all known MHC-class-I-engaging inhibitory receptors but retain expression of 2B4 (and express MHC class I molecules).

The authors then investigated the role of $2 \mathrm{~B} 4$ in an in vivo situation, by looking at the effect of 2B4 on the elimination of syngeneic cells in bone-marrow transplants. Wild-type and $2 b 4^{-/-}$mice were injected with mixed bone marrow containing labelled $\beta_{2}$ m-deficient bone-marrow cells from $\mathrm{C} 57 \mathrm{BL} / 6$ mice, as well as wild-type C57BL/ 6 bone-marrow cells labelled with a different dye. Spleens were isolated 2 days later, and the remaining donor cells were counted. Compared with wild-type mice, $2 b 4^{-/-}$mice showed greater rejection of transplanted $\beta_{2} \mathrm{~m}^{-/-}$cells.

These results show that 2B4-CD48 interactions are an additional system for controlling NK-cell tolerance.

Elaine Bell

(D) References and links ORIGINAL RESEARCH PAPER McNerney, M. E. Guzior, D. \& Kumar, V. 2B4 (CD244)-CD48 interactions provide a novel MHC class I-independent system for NK cell self-tolerance in mice. Blood 3 May 2005 (doi:10.1182/blood-2005-01-0357)

FURTHER READING Kumar, V. \& McNerney, M. E.

A new self: MHC-class--1independent natural-killer-cell self-tolerance. Nature Rev. Immunol. 5, 363-374 (2005)

\title{
A new ligand for TLR11
}

It is now well established that Toll-like receptors (TLRs) are crucial for the recognition of bacteria and viruses, but their roles in innate responses to parasitic infections are less well known. Now, reporting in Science, Yarovinsky et al. identify a ligand derived from the protozoan parasite Toxoplasma gondii that is recognized by TLR11, contributing to resistance to infection with this parasite.

Previously, it has been shown that interleukin-12 (IL-12) is essential for host resistance to T. gondii and that parasite-induced IL-12 production requires the TLR adaptor MyD88 (myeloid differentiation primary-response gene 88 ). So, the authors set out to identify the parasitic component and the TLR that might be involved.

Using gel filtration, the authors fractionated STAg, a soluble extract of the tachyzoite stage of T. gondii, and identified a low-molecularweight protein that stimulated the production of high levels of IL-12 by dendritic cells (DCs). This protein had marked sequence homology to profilin proteins present in other protozoa. Consistent with the known MyD88 dependence of T. gondii-induced IL-12 production, DCs from Myd88 $8^{-/-}$mice had impaired IL-12 responses to the recombinant $T$. gondii profilin.

So far, only two TLRs have been shown to recognize microbial-protein ligands - TLR5 (flagellin) and TLR11 (uropathogenic-bacteriaderived protein) - so the authors tested these TLRs for their ability to recognize T. gondii profilin. Indeed, TLR11 transfectants, but not TLR5 (or other TLR) transfectants, displayed dose-dependent activation of nuclear factor- $\kappa \mathrm{B}$ when stimulated with profilin. Moreover, TLR11-deficient DCs failed to produce IL-12 in response to profilin, whereas DC populations from other TLR-deficient mice had no marked cytokine defects, indicating a role for a profilin-TLR11 interaction in IL-12 production in vitro.

Next, the authors showed that the profilinTLR11 interaction is important for resistance to infection with $T$. gondii, as TLR11-deficient mice developed more brain cysts in the chronic phase of infection than did wild-type animals. This was accompanied by reduced levels of IL-12 and interferon- $\gamma$, as observed for $M y d 88^{-/-}$mice. The fact that the TLR11-deficient mice survived acute infection with T. gondii, unlike $M y d 88^{-/-}$ mice, indicates that other MyD88-dependent TLR-family members might also be involved in resistance to infection with $T$. gondii.

Future studies aim to identify whether T. gondii profilin homologues from other related parasites are also recognized by TLR11 and whether they are important in protective responses.

Lucy Bird

(4) References and links ORIGINAL RESEARCH PAPER Yarovinsky, F. et al. TLR11 activation of dendritic cells by a protozoan profilin-like protein. Science 28 Apr 2005 (doi:10.1126/science. 1109893)

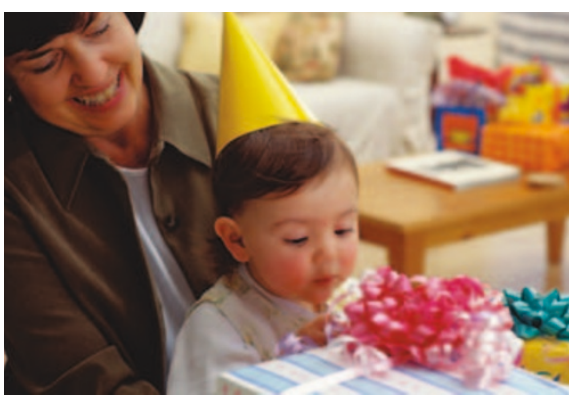

